УДК 517.9

\title{
Impact of Reflexivity on a Dynamics of a Population with Optimal Migration and Global Information Access
}

Michael G. Sadovsky*

Mariya Yu. Senashova ${ }^{\dagger}$

Institute of Computational Modeling SB RAS

Akademgorodok, 50/44, Krasnoyarsk, 660036

Russia

Received 10.05.2015, received in revised form 10.06.2015, accepted 20.07.2015

Comparison of reflexive vs. non-reflexive behaviour is provided, for the dynamics of globally informed beings in a community with optimal migration.

Keywords: population dynamics, optimization migration, global information access, Ferchulst equation. DOI: $10.17516 / 1997-1397-2015-8-3-340-342$

\section{Reflexive-free model of the dynamics of globally informed beings}

We consider a dynamics of spatially distributed communities where being perform optimal relocation of themselves in space; optimality means maximization of net reproduction $[1,2]$. We shall suppose a community to consist of two stations (habitation entity) occupied with two species; migration is stipulated to be a transfer from station to station, exclusively. Besides, the beings are supposed to have global access to information/knowledge on environmental conditions, subpopulation density, transfer cost etc. The aim of the paper is to figure out the impact of reflexivity on the dynamics.

We start from a single species population subdivided into two subpopulations. Let the dynamics of each subpopulation in migration-free case follows Verchult's equation:

$$
N_{t+1}=N_{t}\left(a-b \cdot N_{t}\right) ; \quad M_{t+1}=M_{t}\left(c-d \cdot M_{t}\right)
$$

where $N_{t}$ and $M_{t}$ are the abundances of the subpopulations in the time moment $t$, in station $\mathrm{I}$ and II , respectively; $a$ and $c$ are Malthusian parameters while $b$ and $d$ describe the density dependent regulation. The functions in the parentheses in (1) are the net reproduction, in the corresponding stations.

A migration (between the stations) runs if and only if the living conditions at the immigration station become better than these latter at the habitation station, with respect to transfer cost $p$. The figure $p,(0 \leqslant p \leqslant 1)$ is a probability of a successful transfer from station to station; i. e. the transfer that brings no harm for further reproduction.

The migration from station $\mathrm{I}$ to station II (and from II to I, respectively) starts, if:

$$
a-b N_{t}<p \cdot\left(c-d M_{t}\right) \quad \text { or } \quad c-d M_{t}<p \cdot\left(a-b N_{t}\right) .
$$

*msad@icm.krasn.ru

$\dagger$ msen@icm.krasn.ru

(C) Siberian Federal University. All rights reserved 
The number $\Delta$ of beings migrating from station to station must equalize the inequality (2). Obviously, $\Delta \leqslant N_{t}$ (or $\Delta \leqslant M_{t}$ ). If the migration runs at the time moment $t$, then $\Delta$ is defined by

$$
\vec{\Delta}=\min \left\{N_{t}, \frac{a-b N_{t}+p c-p d M_{t}}{b+p^{2} d}\right\} ; \overleftarrow{\Delta}=\min \left\{M_{t}, \frac{c-d M_{t}+p a-p b N_{t}}{d+p^{2} b}\right\}
$$

So, the model works as following: for each $t$ a migration conditions (2) are checked, and they hold true, the migration fluxes $(3)$ are determined. Then the upgraded abundances $\widetilde{N}_{t}$ and $\widetilde{M}_{t}$ are determined as

$$
\widetilde{N_{t}}=N_{t}+p \Delta, \quad \widetilde{M_{t}}=M_{t}-\Delta ; \quad \text { or } \quad \widetilde{N_{t}}=N_{t}-\Delta, \quad \widetilde{M}_{t}=M_{t}+p \Delta
$$

in dependence on the migration direction. It may be $\Delta_{t}=0$, for some $t$. Finally, the next generation abundances for $t+1$ time moment are calculated according to (1), with upgraded figures $\widetilde{N}_{t}$ and $\widetilde{M}_{t}$. The model $(1-3)$ is irreversible in time: migration is a projection here. It should be stressed, that the model has no reciprocal migration fluxes.

\section{Reflexive model of the dynamics of globally informed beings}

Reflexivity means an ability to foresee the behaviour of a counterparty at the conflict [3]. We stipulate here that each subpopulation is divided into two "subspecies": reflexive $\left(R_{t}\right)$ and reflexive-free $N R_{t}$ beings. Suppose that $N_{0}=N R_{0}^{I}, M_{0}=R_{0}^{I I}$ for $t=0$. Further, the abundances in each station are the sums of these latter of both "subspecies": $N R_{t}^{I}+R_{t}^{I}$ and $N R_{t}^{I I}+R_{t}^{I I}$. The model is to be changed in the following way to define the migration conditions:

$$
a-b\left(N R_{t}^{I}+R_{t}^{I}\right)<p \cdot\left(c-d\left(N R_{t}^{I I}+R_{t}^{I I}\right)\right) \operatorname{orc}-d\left(N R_{t}^{I I}+R_{t}^{I I}\right)<p \cdot\left(a-b\left(N R_{t}^{I}+R_{t}^{I}\right)\right)
$$

A feasibility of a condition in (4) yields the migration flux $\Delta_{N R}$ for reflexive-free subspecies accompanied with the upgrade of abundances $\widetilde{N R_{t}^{I}}$ and $\widetilde{N R_{t}^{I I}}: \widetilde{N R_{t}^{I}}=N R_{t}^{I}-\Delta$, $\widetilde{N R_{t}^{I I}}=N R_{t}^{I I}+p \Delta, \quad(I \rightarrow I I)$ or $\widetilde{N R_{t}^{I}}=N R_{t}^{I}+p \Delta, \widehat{N R_{t}^{I I}}=N R_{t}^{I I}-\Delta,(I I \rightarrow I)$. Then the constraints (4) is checked, for reflexive subspecies, and the migration flux $\Delta_{R}$ is determined together with the relevant upgraded abundances. Finally, the abundances for both subspecies, at the time moment $t+1$ are calculated: $N R_{t+1}^{I}=\widetilde{N R_{t}^{I}}\left(a-b \cdot\left(\widetilde{N R_{t}^{I}}+\widetilde{R_{t}^{I}}\right)\right), \quad R_{t+1}^{I}=\widetilde{R_{t}^{I}}\left(a-b \cdot\left(\widetilde{N R_{t}^{I}}+\widetilde{R_{t}^{I}}\right)\right) ; \quad N R_{t+1}^{I I}=$ $\widetilde{N R_{t}^{I I}}\left(a-b \cdot\left(\widetilde{N R_{t}^{I I}}+\widetilde{R_{t}^{I I}}\right)\right), R_{t+1}^{I I}=\widetilde{R_{t}^{I I}}\left(c-d \cdot\left(\widetilde{N R_{t}^{I I}}+\widetilde{R_{t}^{I I}}\right)\right)$.

\section{Simulations}

We simulated the dynamics with the following parameters: $2 \leqslant a \leqslant 10$ and $2 \leqslant c \leqslant 10$ both with the step equal to $0.16 ; 0.0001 \leqslant b \leqslant 0.0005$ (same for $d$ ) with the step equal to 0.000008 , and $0.1 \leqslant p \leqslant 1$ with the step 0.1 . Each run was as long, as $10^{3}$ generations; initial abundances of all subspecies were equal to $10^{3}$, in both stations. 


\begin{tabular}{|c|c|c|c|}
\hline$p$ & $S_{N}, \%$ & $S_{N R}, \%$ & $\hat{S}, \%$ \\
\hline 0.1 & 31.10642655 & 48.9710452 & 19.92252825 \\
0.2 & 13.52659247 & 65.56794783 & 20.9054597 \\
0.3 & 6.37460658 & 77.29145911 & 16.33393431 \\
0.4 & 4.098623796 & 83.98300407 & 11.91837213 \\
0.5 & 4.335381006 & 86.07933738 & 9.585281614 \\
0.6 & 5.963787304 & 86.8060692 & 7.230143498 \\
0.7 & 8.569575901 & 86.01201558 & 5.41840852 \\
0.8 & 10.47916745 & 86.42967115 & 3.091161406 \\
0.9 & 12.47091839 & 86.483895 & 1.045186614 \\
1.0 & 8.030887804 & 91.78426439 & 0.184847806 \\
\hline
\end{tabular}

So, for every figure of $p$ the set of $50^{4}$ parameters $a, c, b, d$ figures has been used in simulation to calculate the average abundances: $N$ is the average over the generations series over two stations abundance of reflexive-free subspecies, and $N R$ is similar figure for reflexive species. Totally, $10 \times 50^{4}$ trajectories of the model have been simulated, and the average figures have been determined. An advantage of reflexivity has been observed for some parameters combinations, while an opposite situation for other; there were some combinations where advantage of neither strategy has been observed. Table at the left shows this situation: $S_{N}$ is the portion of cases where reflexive-free strategies bring advantage, $S_{N R}$ is the portion of the opposite cases; finally, $\hat{S}$ is the portion of cases with the parity of strategies.

\section{References}

[1] A.N.Gorban, M.G.Sadovsky, Optimal strategy of spatial distribution: Alle's effect, Rus. J. of Gen. Biol., 50(1989), no. 1, 16-21.

[2] M.G.Sadovskii, M.Yu.Senashova, On the problem of modeling reflexive behavior in conflict by an example of biological communities, J. of Appl. and Industr. Math., 8(2014), no. 3, $375-384$.

[3] V.A.Lefebvre, The law of self-reflexion, Reflexive Processes and Control 1(2002), no. 2, 91-99.

\section{Влияние рефлексивного поведения на динамику численности одновидовой популяции глобально информированных особей}

Михаил Г. Садовский
Мария Ю. Сенашова

$\overline{\text { В статье сравниваются модели динамики популяции пространственно распределённой одновидо- }}$ вой популяции глобально информированных особей с рефлексивным поведением и без него.

Ключевые слова: динамика популячии, оптимизачионная миграция, глобальная информированность, уравнение Ферхюльста. 